February 2005

\title{
Two-dimensional Fluorescence Difference Gel Electrophoresis Analysis of the Urine Proteome in Human Diabetic Nephropathy
}

\author{
Kumar Sharma \\ Thomas Jefferson University \\ SoHee Lee \\ Thomas Jefferson University \\ Steven Han \\ Thomas Jefferson University \\ Sungchun Lee \\ Thomas Jefferson University
}

Barbaratersan\&qs âditional works at: https://jdc.jefferson.edu/medfp Thomas Jefferson University

Part of the Medical Genetics Commons

\section{Let us know how access to this document benefits you}

\section{Recommended Citation}

Sharma, Kumar; Lee, SoHee; Han, Steven; Lee, Sungchun; Francos, Barbara; McCue, Peter; Wassell, Richard; Shaw, M. Alexander; and RamachandraRao, Satish P., "Two-dimensional Fluorescence Difference Gel Electrophoresis Analysis of the Urine Proteome in Human Diabetic Nephropathy" (2005). Department of Medicine Faculty Papers. Paper 1.

https://jdc.jefferson.edu/medfp/1

This Article is brought to you for free and open access by the Jefferson Digital Commons. The Jefferson Digital Commons is a service of Thomas Jefferson University's Center for Teaching and Learning (CTL). The Commons is a showcase for Jefferson books and journals, peer-reviewed scholarly publications, unique historical collections from the University archives, and teaching tools. The Jefferson Digital Commons allows researchers and interested readers anywhere in the world to learn about and keep up to date with Jefferson scholarship. This article has been accepted for inclusion in Department of Medicine Faculty Papers by an authorized administrator of the Jefferson Digital Commons. For more information, please contact: JeffersonDigitalCommons@jefferson.edu. 


\section{Authors}

Kumar Sharma, SoHee Lee, Steven Han, Sungchun Lee, Barbara Francos, Peter McCue, Richard Wassell, M. Alexander Shaw, and Satish P. RamachandraRao 


\title{
Two-dimensional fluorescence difference gel electrophoresis analysis of the urine proteome in human diabetic nephropathy
}

\author{
Kumar Sharma ${ }^{1,2}$, SoHee Lee ${ }^{1,2}$, Steven Han $^{1,2}$, Sungchun Lee ${ }^{1,2}$, Barbara Francos ${ }^{1}$, \\ Peter McCue ${ }^{3}$, Richard Wassell ${ }^{4}$, M. Alexander Shaw ${ }^{4}$ and Satish P. RamachandraRao ${ }^{2}$ \\ 1 Center for Diabetic Kidney Disease, Thomas Jefferson University, Philadelphia, PA, USA \\ 2 Dorrance Hamilton Research Laboratories, Thomas Jefferson University, Philadelphia, PA, USA \\ 3 Department of Pathology, Anatomy and Cell Biology, Thomas Jefferson University, Philadelphia, PA, USA \\ 4 Proteomics Core Facility, Kimmel Cancer Center, Thomas Jefferson University, Philadelphia, PA, USA
}

Correspondence: Dr. Kumar Sharma, Thomas Jefferson University,

Suite 353, Jeff Alumni Hall, 1020 Locust Street, Philadelphia,

PA 19107, USA

E-mail: kumar.sharma@jefferson.edu

Fax: 11-215-923-7212

\begin{abstract}
Urinary proteins may provide clues regarding pathogenesis of kidney disease as well as providing markers of disease activity. We employed two-dimensional differential in-gel electrophoretic analysis (2-D DIGE) to assess multiple urine samples in patients with diabetic nephropathy. Patient samples were collected as timed overnight collections. All the patients had longstanding diabetes, impaired renal function, and overt proteinuria. Control and patient urinary protein were analyzed by 2-D DIGE and DeCyder analysis. Ninety-nine spots were significantly regulated in the urine proteome of the diabetic samples, with 63 up- and 36 down-regulated. One spot corresponding to a pl 5-6 and a molecular weight between 45 and 66 kDa was consistently upregulated by 19 -fold across individuals in the diabetic group. Surfaceenhanced laser desorption/ionization-time of flight analysis of in-gel tryptic digest of this spot identified this protein as alpha 1 antitrypsin (AAT). ELISA of urine samples from a separate group of patients and controls confirmed a marked increase of AAT in diabetic patients. Immunostaining of human diabetic kidneys revealed upregulation of AAT in areas of renal fibrosis. In conclusion, we developed a method to analyze numerous urine samples from patients and allowed for detection and identification of regulated urine protein spots.
\end{abstract}

\section{I ntroduction}

The incidence of diabetic nephropathy continues to increase and now accounts for almost $50 \%$ of all new cases of endstage renal disease [1]. This rise is noted despite an increased awareness of early detection of microalbuminuria as well as the benefits of good glycemic [2] and blood pressure control with renin-angiotensin inhibition [3]. Several trials have demonstrated improved mortality and delayed progression of nephropathy with the use of ACE-inhibitors and angiotensin II receptor antagonists in diabetics [3-6]. However, many patients progress to end-stage renal disease despite adequate therapy. In order to identify patients who will progress despite therapy, new biomarkers are needed in the patient population with declining renal function. Although analysis of kidney tissue may provide clues to determine which patients 
may progress, current clinical practice does not allow for routine kidney biopsies as the procedure is invasive and may suffer from sampling error. The use of plasma or serum may provide clues to differentiate patients who progress from those who do not, however, blood samples may not reflect renal disease specifically.

Recently, the development of methods for comparing urinary proteomes has been demonstrated to identify proteins, or protein patterns, that may serve as biomarkers or identify new pathogenetic paradigms for human renal diseases $[7,8]$. The urinary proteomes of normal and pathologic states have been compared using various techniques such as differential analysis of proteins separated by 2-D PAGE, LC, SELDI-TOF MS, and isotope-coded affinity tags [7-10]. Similar studies have not been reported in diabetic patients. However, there remain difficulties with the above approaches. Quantitative analysis of the urinary proteins from numerous 2-D gels may be difficult to compare, and gel-gel variation will further complicate the analysis. The SELDITOF MS approach is useful for the urinary protein patterns, but definitive identification of differentially regulated proteins is challenging.

Recently, 2-D DIGE using fluorescently labeled proteins has been described to obviate gel-gel variability and allow for comparison of two separate sources of proteins in one gel [11]. In the present study, we compared proteomes of the urine samples from diabetic patients with impaired renal function with urines from normals using the 2-D DIGE approach to identify biomarkers associated with diabetic nephropathy. We identified the most highly abundant upregulated spot by MS analysis as alpha 1 antitrypsin (AAT). Up-regulation was validated with ELISA from distinct urine samples of patients with diabetic nephropathy. Furthermore, immunostaining of AAT in human kidney sections with diabetic nephropathy suggested novel pathobiologic pathways by which AAT may contribute to a diabetic nephropathy.

\section{Materials and methods}

\subsection{Patient selection}

The patients in this study were recruited as part of an ongoing placebo-controlled study in diabetic nephropathy. The inclusion criteria were as follows: type 1 or 2 diabetes, age $>18$, glomerular filtration rate (GFR) between 20 and $75 \mathrm{~mL} / \mathrm{min} / 1.73$ $\mathrm{m}^{2}$, history of proteinuria ( $300 \mathrm{mg} / 24 \mathrm{~h}$ or albumin positive by dipstick), and blood pressure controlled to 140/90 with angiotensin converting enzyme inhibitor and/or angiotensin receptor antagonist, if tolerated. The exclusion criteria were as follows: inability to give informed consent, pregnant, breast feeding or lactating, hematologic disease, malignancy, liver disease, viral hepatitis, and $\mathrm{HIV}^{+}$, on immunosuppressive medication, other known renal disease besides diabetic nephropathy by history, congestive heart failure (NYHA class III or worse), history of recent myocardial infarction or unstable angina within past 6 months, and history of peptic ulcer within 6 months. The control samples were healthy male and female volunteers with no known past medical history and who had taken no medications within 2 wk of urine collection. An institutional review board approval for the study was obtained at Thomas Jefferson University and all the patients were recruited at Thomas Jefferson University Hospital. 


\subsection{Sample preparation}

For DIGE analysis, the urine samples were collected from three diabetic males and five healthy male volunteers. Diabetic male samples were compared with control male samples as the urinary proteome may vary with gender [10]. Samples from controls were confirmed negative for proteinuria or hematuria by dipstick (Multistix 10 SG; Bayer, Elkhart, IN, USA). Twenty milliliters of patients' first morning void urine was collected with protease inhibitor (Complete Mini; Roche) and stored at $-80^{\circ} \mathrm{C}$ until further processing to prevent protein degradation. The samples from the male controls were pooled together to be processed as a standard of comparison among the various patient samples.

\subsection{Protein isolation}

Urine samples were processed in $5 \mathrm{~mL}$ increments and centrifuged at $3000 \times \mathrm{g}$ for 5 min. The supernatant was filtered through $0.22 \mu$ filter paper prior to precipitation of urine protein by adding equal volume of acetone at $4^{\circ} \mathrm{C}$ for $10 \mathrm{~min}$. Samples were centrifuged at $12000 \times \mathrm{g}$ for $5 \mathrm{~min}$ at $4^{\circ} \mathrm{C}$. The resulting pellets were resuspended in $8 \mathrm{~m}$ urea and $4 \%$ CHAPS. Protein concentration was determined using the GE Healthcare Quant Kit (Piscataway, NJ, USA). Samples were stored at $-80^{\circ} \mathrm{C}$ in $100 \mu \mathrm{g}$ protein aliquots until further processing.

\subsection{Fluorescent tagging}

Three DIGE gels were run, each comparing the urine proteome of a unique diabetic patient to the urine proteome in normals (see Table 1 ). The samples were brought to $\mathrm{pH}$ of 8-8.5 with $1 \mathrm{M} \mathrm{NaOH}$ solution to optimize fluorescent tagging. For each gel, 50 $\mu \mathrm{g}$ of protein of normal or patient samples were added to $400 \mathrm{pmol}$ of Cy3 or Cy5, and allowed to incubate on ice for $30 \mathrm{~min}$. Additionally, for each gel, $50 \mathrm{\mu g}$ of an internal standard comprising equal amounts of normal and patient protein, was labeled with Cy2. The labeling reaction was quenched by addition of $1 \mu \mathrm{L}$ of $10 \mathrm{~m} \mathrm{M}$ lysine and subsequently incubated on ice for $15 \mathrm{~min}$. For each gel, these three samples (internal standard, patient, and normal) were pooled prior to 2-DE.

Table 1. Experimental design of DIGE gels

\begin{tabular}{llll}
\hline & Gel 1 & Gel 2 & Gel 3 \\
\hline Cy2 & Internal standard & Internal standard & Internal standard \\
Cy3 & Normal & Patient 2 & Normal \\
Cy5 & Patient 1 & Normal & Patient 3 \\
\hline
\end{tabular}

\subsection{IEF}

For each gel, $18 \mathrm{~cm}, \mathrm{pH} 3-10$ IPG strips were actively rehydrated at $30 \mathrm{~V}$ for $12 \mathrm{~h}$ in $350 \mu \mathrm{L}$ of sample, using an IPGphor (GE Healthcare). Once rehydration was complete, samples were focused at $500 \mathrm{~V}$ for $1 \mathrm{~h}, 1000 \mathrm{~V}$ for $1 \mathrm{~h}$, and finally $8000 \mathrm{~V}$ for $6 \mathrm{~h}$. Immediately after completion, IPG strips were either stored at $-80^{\circ} \mathrm{C}$ until further analysis or immediately processed for separation by SDS-PAGE. 


\subsection{SDS-PAGE}

In order to eliminate disulfide bonds in the focused proteins in preparation for the second dimension, IPG strips were incubated for $15 \mathrm{~min}$ in equilibration buffer I, which consisted of $0.375 \mathrm{M}$ Tris/ $\mathrm{HCl} \mathrm{pH} \mathrm{8.8,6} \mathrm{mol} / \mathrm{L}$ urea, 2\% SDS, $20 \%$ glycerol, and $13 \mathrm{mM}$ DTT. The IPG strips were then soaked in equilibration buffer II for an additional 15 min to alkylate the sulfhydryl groups. Buffer II was identical to buffer I with the exception of $2.5 \%$ iodoacetamide instead of DTT. The strips were then embedded in $0.7 \% \mathrm{w} / \mathrm{v}$ agarose on top of $12.5 \%$ acrylamide slab gels. Second dimension separations were performed on a DALT6 platform (GE Healthcare). IPG strips were run at $2 \mathrm{~W} / \mathrm{gel}$ for $30 \mathrm{~min}$, followed by $20 \mathrm{~W} / \mathrm{gel}$ until the dye front reached the bottom of the gel. The gels were rinsed in deionized water and were scanned using the DIGE-enabled Typhoon Scanner (GE Healthcare).

\subsection{Staining and DIGE analysis}

After the images were scanned, GE Healthcare DeCyder software 5.01 was utilized for a differential gel analysis. The 2-D image of the gel from each diabetic patient was compared with that of the normal sample via the initernal standard sample. Specifically, the protein expression analysis was performed for each of the three gels in parallel using the DIA module of DeCyder using a value of 1000 as the initial estimate of protein spots present. DIA analysis allows for the direct comparison of intensities of specific protein spots between different samples within the same gel. In this case, protein intensities were compared between the urine proteomes of specific diabetic patients and a normal urine proteome. These DIA analyses were collated into a single analysis using the BVA module of DeCyder, and final values for the expression ratio of specific protein spots between diabetic and normal patients were determined.

\subsection{MS and PMF}

Protein identification was performed via PMF using a SELDI-TOF mass spectrometer (Ciphergen Biosystems). The gels were silver-stained in order to visualize spots. Formaldehyde was used instead of glutaraldehyde to avoid interference with MS. The silver-stained spots of interest were manually cut from the gel, tryptically digested, and prepared on an H4hydrophobic chip using a matrix of CHCA. Angiotensin I (12965.4 Da) and beta-endorphin (3465.0 Da) were used as internal calibrants. The protein identification of peptide fragments was performed by using the ProFound search engine (Rockefeller University) and the MASCOT software as a secondary check. The National Center for Biotechnology Information (NCBI) protein database was restricted to mammalian entries in the preliminary rounds of analysis and to Homo sapiens in the subsequent round of analysis. The peptides were assumed to be an average mass, oxidized at methionine residues, and carbamidomethylated at cysteine residues. Up to one missed trypsin cleavage and a mass tolerance error of \pm $0.6 \mathrm{Da}$ was allowed for matching peptide mass values. Criteria for protein confirmation included a statistically significant Z-score with a probability of 1.0 , at least $25 \%$ sequence coverage, and the correspondence of the matched peptides with the main peptide peaks of the mass spectrum.

\subsection{ELISA}

In order to validate the results of DIGE analysis and to confirm that urine levels of AAT were elevated in diabetic nephropathy, we performed ELISA on urines from ten 
distinct male diabetic patients, nine female diabetic patients, ten normal males, and ten normal females. An ELISA kit designed for human AAT (ALPCO Diagnostics, Windham, $\mathrm{NH}$, USA) was used. Urine diluted 50 -fold with wash buffer was incubated with polyclonal rabbit antibodies immobilized to the surface of the microtiter wells for $1 \mathrm{~h}$ at room temperature. Wells were aspirated and washed with buffer five times. The samples were incubated with $100 \mathrm{~mL}$ peroxidase-labeled anti-AAT for $30 \mathrm{~min}$ at room temperature and washed five times with buffer. Ten microliters of substrate tetramethylbenzidine was added and incubated for 10-20 min at room temperature. Fifty microliters stop solution was added to stop the reaction, which turned the color from blue to yellow. Absorption was measured at 450 and $620 \mathrm{~nm}$ wavelength with a SPECTRAMax 250 microplate reader (Molecular Devices, Sunnyvale, CA, USA). AAT concentrations were determined from a standard curve generated from the standards supplied with the kit. The measurement of urine creatinine was done by biosensor assay using a NOVA 16 chemistry analyzer. A statistical analysis of ELISA assays of urine AAT was done with analysis of variance (ANOVA). $\quad p<0.05$ was considered statistically significant. The data were reported as mean \pm SEM.

\subsection{I mmunostaining}

Immunohistologic staining was performed on both normal $(n=5)$ and diabetic human kidney sections $(n=10)$ as well as on normal human liver sections for positive and negative controls. Human kidney tissue was obtained from archived paraffin-embedded samples or from discarded specimen from nephrectomy specimens. IRB approval was obtained for procurement of kidney specimens. Three micrometer-thick human kidney sections were deparaffinized and rehydrated. Antigen retrieval was done by incubating in Microwave Citra Plus solution (Biogenex, San Ramon, CA, USA) at $60 \%$ power for $10 \mathrm{~min}$. Endogenous tissue peroxidase activity was suppressed by an incubation in $0.03 \% \mathrm{H}_{2} \mathrm{O}_{2}$ at room temperature for 10 min. Sections were then incubated with mouse monoclonal anti-AAT antibody specific for human AAT (Abcam, Cambridge, MA, USA) at 1:600 for $1 \mathrm{~h}$ at room temperature. The negative control slide with normal human liver was incubated with secondary antibody alone. The slides were washed with Tris buffer before incubation with peroxidase-labeled polymer (DAKO, Carpinteria, CA, USA), and immunoreactive AAT was detected with Chromogen $3^{\prime} 3^{\prime}$ diaminobenzidine for $5 \mathrm{~min}$. All the sections were counterstained with hematoxylin. The specimens were examined by our renal pathologist (P.M.).

\section{Results}

\subsection{Differential gel electrophoresis and PMF}

The clinical data on the patients' samples used for DIGE analysis is presented in Table 2. An overlaid visualization of the protein spot patterns of urine samples from a representative diabetic sample and the normal sample exhibited marked variation (Fig. 1). The DeCyder analysis revealed 289 protein spots that were present in all three gels. Of these, 99 spots exhibited statistically significant differences in protein expression between the diabetic and normal urine samples (63 spots increasing in the diabetic samples and 36 decreasing). A difference was deemed statistically significant if it exhibited a Student's t-test value of 0.005 or less. Of these changing spots, one highly abundant spot in particular, corresponding to a pl 5-6 and with a molecular mass between 45 and $66 \mathrm{kDa}$, was up-regulated 19-fold in the urine of diabetic versus normal males (Fig. 2). In-gel tryptic digestion and SELDI-TOF 
analysis was performed on this spot. The SELDITOF mass spectra of the tryptic digest are represented in Fig. 3. Using the ProFound search engine, this protein was identified as AAT. Of 34 measured peptides, 22 sequences matched those found in AAT. Sequence coverage was $52 \%$. In addition to this protein spot, identification of additional changing proteins via PMF is ongoing.

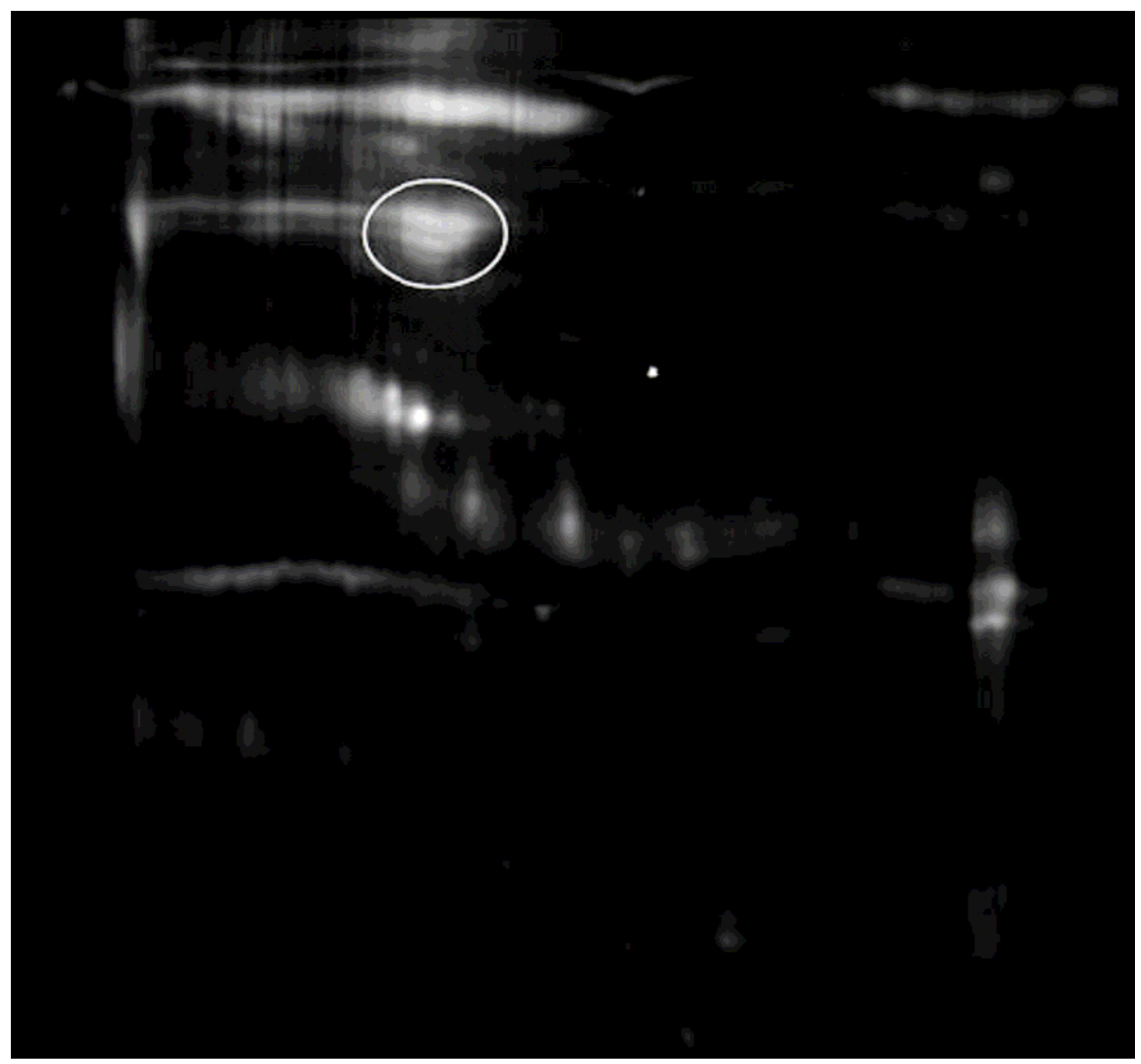

Figure 1. Overlaid 2-D gel images of normal (green) and patient (red) protein spot patterns. Note prominent red spot (circled) below albumin streak, indicating a protein that is highly up-regulated in the patient sample. 
Table 2. Clinical and demographic data for patients in whose urine proteomes were analyzed

\begin{tabular}{|c|c|c|c|c|c|}
\hline & Patient 1 & Patient 2 & Patient 3 & Mean & SEM \\
\hline Age & 58 & 82 & 49 & 63 & 9.85 \\
\hline Sex & Male & Male & Male & $\mathrm{N} / \mathrm{A}$ & N/A \\
\hline Type I or type II diabetes mellitus & I & II & $\mathrm{I}$ & N/A & $\mathrm{N} / \mathrm{A}$ \\
\hline Race & Caucasian & African-American & Caucasian & $\mathrm{N} / \mathrm{A}$ & $\mathrm{N} / \mathrm{A}$ \\
\hline Taking ACEI/ARB & Yes & Yes & Yes & N/A & $\mathrm{N} / \mathrm{A}$ \\
\hline Taking HMGcoA reductase inhibitor & Yes & Yes & Yes & $\mathrm{N} / \mathrm{A}$ & $\mathrm{N} / \mathrm{A}$ \\
\hline Taking thiazolidinedione & No & No & No & N/A & $\mathrm{N} / \mathrm{A}$ \\
\hline Serum creatinine (mg/dL) & 2.5 & 1.6 & 1.4 & 1.83 & 0.13 \\
\hline GFR $\left(\mathrm{mL} / \mathrm{min} / 1.73 \mathrm{~m}^{2}\right)$ & 44.194 & 47.918 & 75.449 & 55.85 & 3.92 \\
\hline Systolic BP (mm hg) & 139 & 138 & 131 & 136 & 1 \\
\hline Urine albumin $(\mathrm{mg} / \mathrm{g} \mathrm{Cr})$ & 306.8 & 1762.1 & 422.08 & 830.34 & 185.6 \\
\hline Hemoglobin A1C (\%) & 7.4 & 8.8 & 7.4 & 7.87 & 0.19 \\
\hline
\end{tabular}

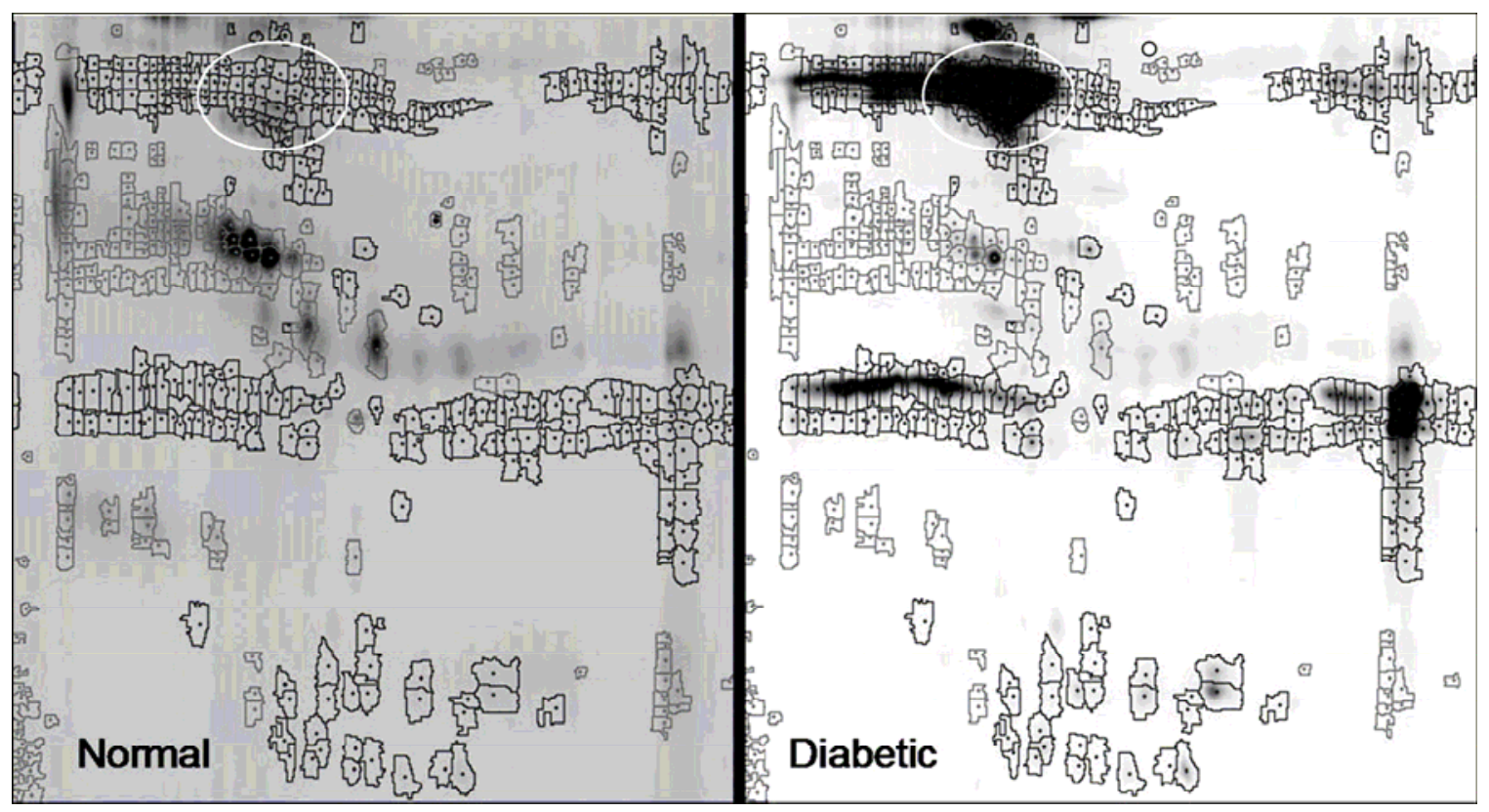

Figure 2. DIGE analysis of region below albumin streak. Blue spots represent proteins greater than three times proportionately higher in diabetics than normals. Red spots represent proteins greater than three times proportionately lower in diabetic sample than pooled normals. One spot in particular was upregulated $>19$-fold among diabetic samples (circled). 


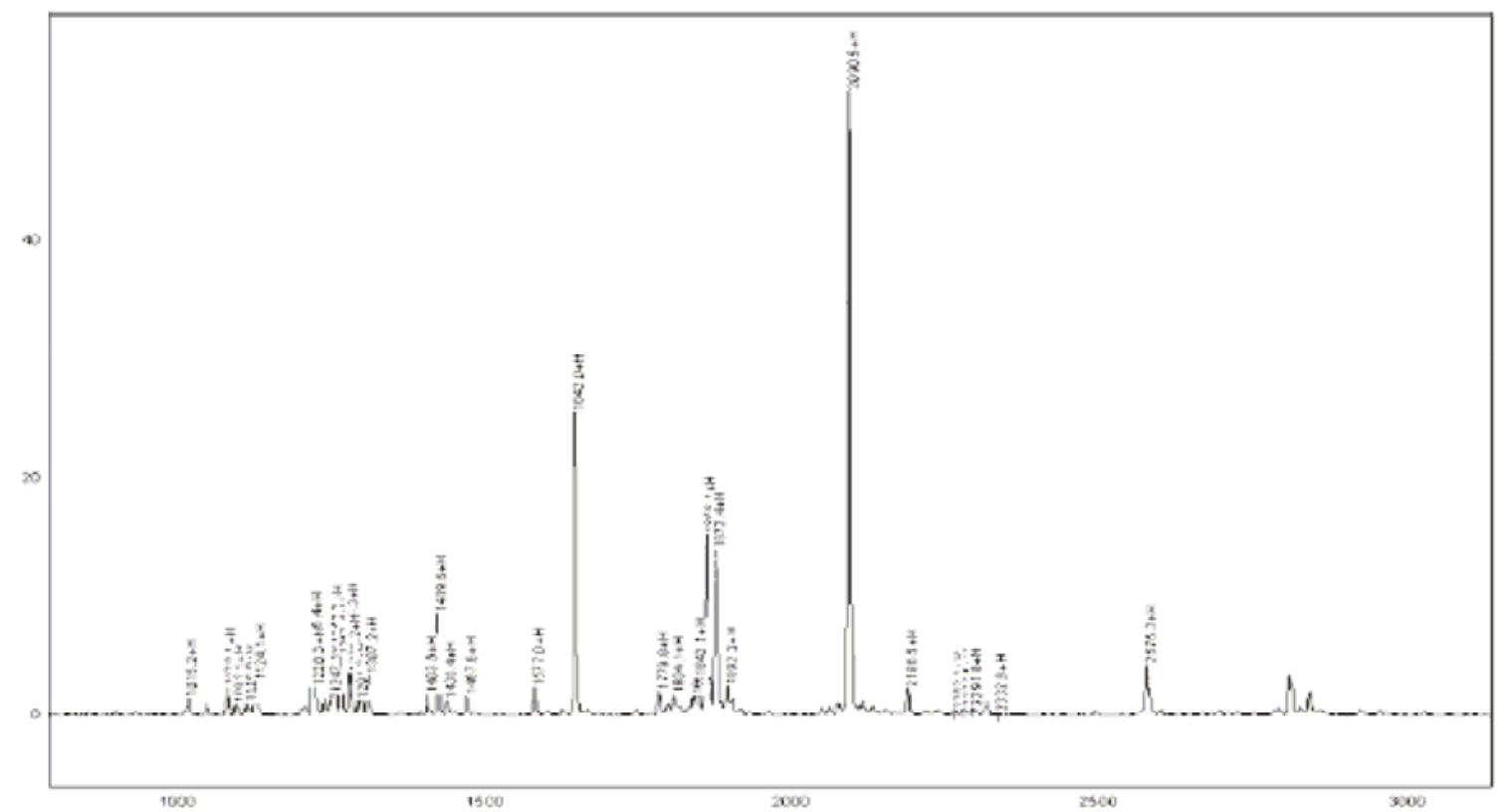

Figure 3. MS analysis after tryptic digestion of spot of interest from 2-D SDS-PAGE. This protein spot was identified as AAT by MS via PMF with greater than $99 \%$ confidence.

\subsection{ELISA}

In order to confirm that AAT was indeed increased in urine of patients with diabetic nephropathy, we performed ELISA on urine obtained from 19 separate diabetic male and female patients with proteinuria and 20 normal volunteers. The clinical characteristics of the 19 diabetic patients are shown in Table 3. Urine concentrations were divided by urine creatinine concentration to account for individual differences in urine concentration. Mean urine level of AAT per $\mathrm{mg}$ of urine creatinine in diabetics were increased by over 30-fold (867.49 \pm 257.32 (diabetics) vs. $27.31 \pm 4.78$ $\mathrm{mcg} / \mathrm{mg} \mathrm{Cr}$ (controls), $\mathrm{p}=0.0048$ ) (Fig. 4). The measurements of urinary AAT fell on the linear portion of the ELISA standard curve. 


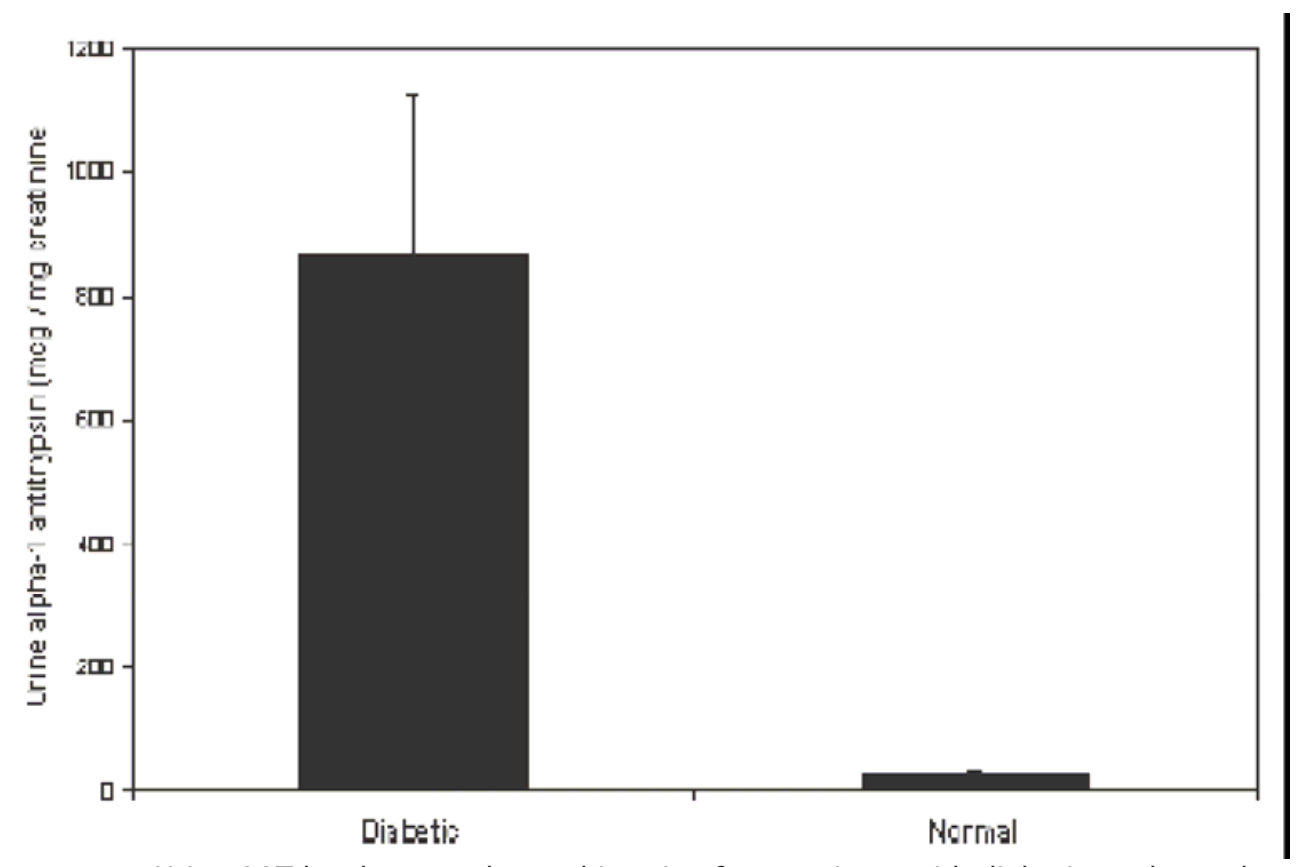

Figure 4. Urine AAT levels were elevated in urine from patients with diabetic nephropathy. AAT (corrected for urine creatinine) in diabetic urines was highly significant compared to controls, $p=0.005$.

Table 3. Clinical and demographic data for patients in whose urine AAT ELISA assays were performed

\begin{tabular}{ll}
\hline Patient characteristics & \\
& Patients $(\mathrm{n}=19)$ \\
\hline Age (years) & 59.3 \\
Females & 9 \\
Males & 10 \\
Type I diabetics & $6(31.6 \%)$ \\
Type II diabetics & $13(68.4 \%)$ \\
Blacks & $5(26.3 \%)$ \\
Caucasians & $14(73.7 \%)$ \\
Patients taking ACE-inhibitor or angioiotensin receptor antagonist & $18(94.7 \%)$ \\
Patients on HMGCoA reductase inhibitor & $16(84.2 \%)$ \\
Patients on thiazolidinedione & $5(26.3 \%)$ \\
Mean plasma creatinine (mg/dL) & $1.99( \pm 0.19)$ \\
Mean glomerular filtration rate (mL/min/1.73 $\left.\mathrm{m}^{2}\right)$ & $47.50( \pm 4.26)$ \\
Mean systolic blood pressure (mm hg) & $129.32( \pm 2.42)$ \\
Mean urine albumin per urine $\mathrm{creatinine}(\mathrm{mg} / \mathrm{g})$ & $427.24( \pm 127.85)$ \\
Mean glycosylated hemoglobin $(\%)$ & $7.27( \pm 0.32)$ \\
\end{tabular}

\subsection{I mmunohistochemistry}

Immunostaining of the sections of human kidneys from patients with diabetic nephropathy and normals showed a distinct staining pattern for AAT (Fig. 5). Areas of increased staining of AAT appeared to be adjacent to the areas of fibrosis in glomerular, interstitial, and perivascular regions from the diabetic samples. In addition there was distinct staining of the luminal border of the proximal tubules of the diabetic kidney in a pattern not seen in the normal kidney. To further demonstrate that human kidneys synthesized AAT, mRNA for AAT was found to be expressed in a normal human kidney by Affymetrix Chip analysis (P.M., unpublished data). 


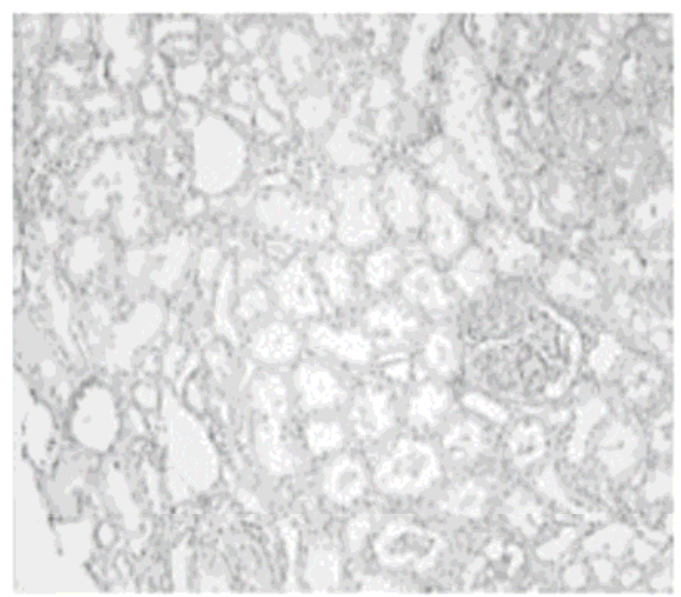

A

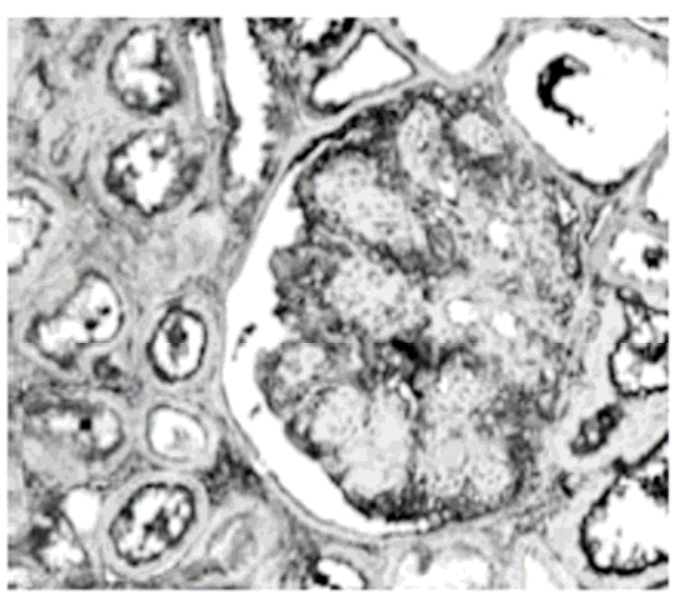

C

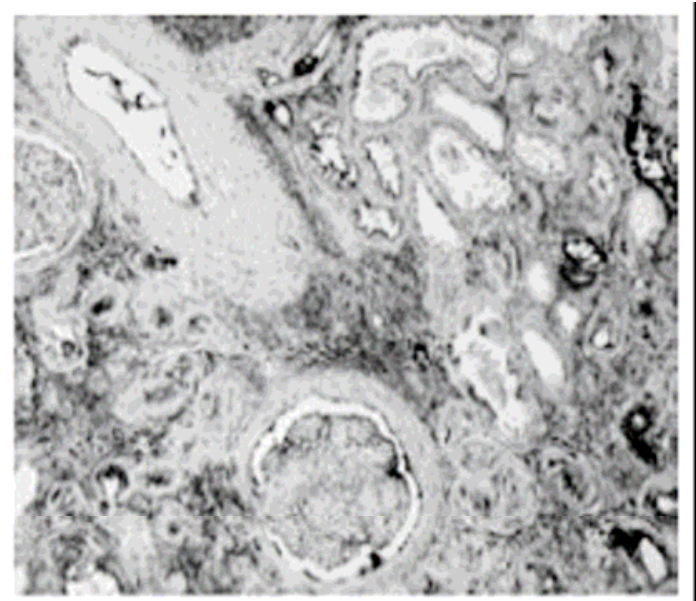

B
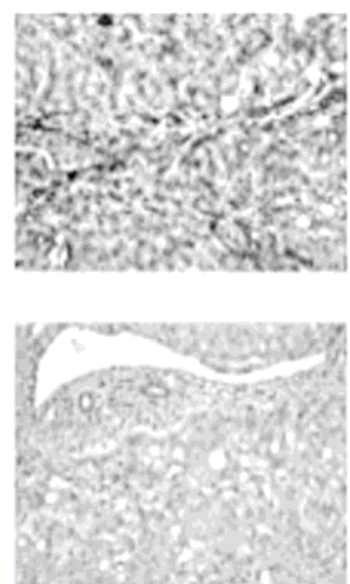

D

Figure 5. Immunostaining of AAT of normal human kidney (A) and kidney showing an advanced diabetic nephropathy (B). There is a marked increase in AAT immunoreactivity at sites of periglomerular fibrosis, intraglomerular sclerosis, perivascular fibrosis, and interstitial fibrosis. A magnification 200x. A higher magnification image (400x) shows AAT in diabetic glomerulosclerosis and proximal tubules (C). A positive control for AAT in normal human liver section (upper panel, D) and a negative control stain using only secondary antibody (lower panel, D), magnification 200x.

\section{Discussion}

The use of 2-D DIGE analysis to detect differences between the disease states and normal has great potential in finding biomarkers of disease and giving clues to the pathogenesis of disease from various tissue samples. In the present study, we report the use of 2-D DIGE for analyzing differences in the urine proteomes from patients with advanced diabetic nephropathy. Using this method we identified AAT as the most highly up-regulated protein (apart from albumin and IgG) in urine from patients with diabetic nephropathy and validated our findings by ELISA of urines. A novel role for AAT is suggested based on its distribution in the diseased kidney.

Urine samples present several unique challenges to proteomic analysis. Marked degradation of proteins can occur due to improper storage. The use of protease 
inhibitors was found to preserve the full complement of proteins present in urines when stored at $-20^{\circ} \mathrm{C}$. Protein degradation may be minimized with storage at $-80^{\circ} \mathrm{C}$, although the combination of protease inhibitors and $-80^{\circ} \mathrm{C}$ storage is likely optimal for long-term storage. It has been recognized that urine protein levels are altered with timing of the collection as well as the diet. To obviate these changes we only analyzed urine samples from first morning, fasting collections. The urine proteome can also vary depending on gender, thus necessitating that the control sample be matched for gender. Use of DIGE offers major advantages to the use of separate comparative gels or a SELDI chip-based approach. With the latter it is difficult to characterize and identify differentially regulated proteins, and with the former approach the statistical analysis becomes very difficult as there are many variables to account for. With DIGE, the advantage of running multiple samples on the same gel offers greater confidence that the proportionate differences reported by DeCyder are valid and reproducible. In our study, the differential regulation of the most highly abundant up-regulated spot across three separate gels was confirmed by independent ELISA analyses from separate urine samples.

AAT is a 394 amino acid $52 \mathrm{kDa}$ acute-phase glycoprotein, which is produced predominantly in the liver and secreted in the vascular bed. It is a member of the serine protease inhibitor (SERPIN) family, the predominant protease inhibitors in mammals. AAT is the natural inhibitor of neutrophil elastase as well as other proteases. Over 90 alleles have been identified. The major physiological function of neutrophil elastase is in host-defense. Neutrophil elastase also degrades a range of substrates including elastin and other extracellular matrix proteins such as collagen, proteoglycan, fibronectin, complement receptors, thrombomodulin, lung surfactant, and several growth factors [12]. It is interesting to note that up-regulation of AAT would lead to inhibition of elastase and thus may contribute to accumulation of matrix molecules in diabetic nephropathy. A recent description that elastin is upregulated in diabetic kidneys would fit with this model [13].

AAT has previously been identified to increase in the urine of patients with kidney disorders including diabetic nephropathy [14]. However, it was considered to be a marker of glomerular leak of serum proteins, similar to albumin. Subsequent studies have found variable plasma levels of AAT in diabetic patients $[15,16]$ and a recent study implicated AAT in possible cardiovascular complications of diabetes [17]. However, there have been no recent studies that have examined urinary AAT as a marker of progressive diabetic nephropathy and considered renal AAT to be of possible pathobiologic signficance. Our studies suggest that a local production of AAT in the kidney may contribute to the increased levels found in the urine and may be associated with the regulation of inflammation and matrix accumulation. Longitudinal studies will need to be performed to establish a role for AAT as a biomarker.

The limitations of our study include the small sample size, and the presence of urinary albumin and IgG. The latter leads to streaking of the gels, and albumin degradation products may account for a large number of differentially regulated spots. A repeat analysis with greater number of samples and albumin/IgG removal prior to analysis will likely reveal additional dysregulated protein spots. In conclusion, we optimized procedures for sample preparation and employed DIGE analysis to allow for straightforward analysis of data obtained from urine proteomics for a common disease condition. Further analysis of additional protein spots using similar approaches and albumin/IgG removal will likely lead to the identification of 
additional novel targets that may be involved in disease pathogenesis or serve as biomarkers.

This study was funded in part by the NIH-NIDDK (R01 DK063017 to K.S.). We would like to acknowledge the contribution of Magdalena Potoczek for performing the immunostaining studies.

\section{References}

1. System, U. S. R. D., Division of Kidney, Urologic, and Hematologic Diseases National Institute of Diabetic and Digestive Kidney Disease, NIH 2000.

2. The Diabetes Control and Complications Trial, Kidney Int. 1995, 47, 17031720.

3. Giovanni, G. F., Strippoli, M. C., Deeks, J. J., Francesco, P. S., Craig, J. C., Br. Med. J. DOI 10.1136/bmj. 38237.585000.7C.

4. Lewis, E. J., Hunsicker, L. G., Bain, R. P., Rohde, R. D., N. Engl. J. Med. 1993, 329, 1456-1462.

5. Lewis, E., Hunsicker, L., Clarke, A., Berl, T. et al., N. Engl. J. Med. 2001, 345, 851-860.

6. Brenner, B., Cooper, M., N. Engl J. Med. 2001, 345, 861-869.

7. Schaub, S., Rush, D., Wilkins, J., Gibson, I. W. et al., J. Am. Soc. Nephrol. 2004, 15, 219-227.

8. Cutillas, P., Chalkley, R., Hansen, K., Cramer, R. et al., Am. J. Physiol. Renal Physiol. 2004, 287, F353-F364.

9. Schaub, S., Wilkens, J., Weiler, T., Sangster, K. et al., Kidney Int. 2004, 65, 323-332.

10. Thongboonkerd, V., McLeish, K. R., Arthur, J.M., Klein, J. B., Kidney Int. 2002, 62, 1461-1469.

11. Unlu, M., Morgan, M., Minden, J., Electrophoresis 1997, 18, 2071-2077.

12. Sun, Z., Yang, P., Lancet Oncol. 2004, 5, 182-190.

13. Thongboonkerd, V., Barati, M., McLeish, K., Benarafa, C. et al., J. Am. Soc. Nephrol. 2004, 15, 650-662.

14. Aronoff, S., Schnider, S., Smeltzer, J., Mackay, W. et al., Diabetes 1981, 30, 656-663.

15. Bristow, C. L., Di Meo, F., Arnold, R. R., Clin. Immunol. Immunopathol. 1998, 89, 247-259.

16. Murakami, T., Komiyama, Y., Egawa, H.,Murata, K., Diabetes 1993, 42, 233238.

17. Talmud, P. J., Martin, S., Steiner, G., Flavell, D. M. et al., Arterioscler. Thromb. Vasc. Biol. 2003, 23, 644-649. 